\title{
Distinction of Closely Spaced Targets using Pseudo Resolution Technique
}

\author{
Amit Agarwal \\ E\&C Dept. SMIT; Majitar \\ Sikkim; INDIA-737136
}

\author{
Debasish Bhaskar \\ E\&C Dept. SMIT; Majitar \\ Sikkim; INDIA-737136
}

\author{
Rabindranath Bera \\ E\&C Dept. SMIT; Majitar \\ Sikkim; INDIA-737136
}

\begin{abstract}
The radar range resolution depends on signal bandwidth thereby detection of multiple closely spaced scattered points depends on bandwidth. To resolve this method of pseudo resolution is introduced which exploits the bandwidth allocation and increases the resolution of the overlapping scattered point (target/multipath). This resolution can be achieved by increasing the bandwidth of the received signal at receiver by stuffing zeros in between the samples received. This is why such bandwidth allocation is referred to as virtual bandwidth. The entire method is called Pseudo resolution.
\end{abstract}

\section{General Terms}

Multipath effects, Bandwidth exploration, Radar processing, target realization, intelligent transport system, collision avoidance and warning system.

\section{Keywords}

pseudo resolution, adaptive bandwidth, Multipath.

\section{INTRODUCTION}

Radar [1] is an object-detection system which uses radio waves to determine the range, altitude, direction, or speed of objects. It can be used to detect vehicles, terrain etc. The radar antenna transmits pulses of radio waves or microwaves which bounce off any object in their path. The object returns a tiny part of the wave's energy to antenna which is usually located at the same site as the transmitter [2].

In a heavy traffic road condition there is maximum chances of occurrence of collision between vehicles and it mainly occurs between closely spaced vehicles which may be few centimeters apart, so to avoid this tragedy, collision avoidance and warning system [3] is introduced which performs detection of how far another vehicle is located and communication is made to the driver to avoid collision. But when vehicles are too closely spaced then there may be occurrence of multipath's from different vehicles or road side structures, which becomes difficult for the system to realize and it may perform faulty observation and causes collision, so to avoid such type of faulty decision the method of pseudo resolution can be implemented for closely spaced targets.[4]

The received signal at the receiver end consists of LOS and multipath echoes [5] from valid targets caused by ground reflection, atmospheric ducting or ionosphere reflection [6]. This multipath type is especially bothersome since it appears to move and behave like other normal targets of interest. The radar may try to unify the targets, reporting the target at an incorrect height, or eliminating it on the basis of jitter or a physical impossibility.
The received LOS and multipath Signal are overlapped with each other so it becomes difficult to distinguish. To resolve this problems pseudo resolution method is introduced.[7]

By increasing bandwidth, realization of the LOS and Multipath may be easier. But due to limited available bandwidth it becomes difficult to increase bandwidth as required, so to realize some of limiting case in which signals becomes difficult to realize, so pseudo realization method comes into play.

\section{EXPERIMENTAL SETUP}

\subsection{Generation of LOS \& Multipath through AWG:}

In AWG (Arbitrary Waveform Generator) the signal generated for second target is considered as multipath in AWG RFXpress.

LOS and Multipath signal is generated using AWG (Arbitrary Waveform Generator) RFXpress. The delay vector and gain vector are designed through the API provided within RFXpress of AWG (They are tabulated in Table: 1). Generated LOS is a 13 Bit pulsed Barker code having a duty cycle $6 \%$ (i.e., $\mathrm{ON}$ time $=32.5 \mathrm{~ns}$ and $\mathrm{OFF}$ time $=$ $509.1667 \mathrm{~ns}$ ). The base-band bandwidth has been configured as $400 \mathrm{MHz}$ (i.e., Barker code [8] chip width $=2.5 \mathrm{~ns}$ ). Also 1 Multipath has been generated through the same RFXpress API available in AWG. The Multipath is delayed by $6 \mathrm{~ns}, 5 \mathrm{~ns}, 4 \mathrm{~ns}$ $\& 3 \mathrm{~ns}$ respectively with respect to LOS. So, for 4 cases of delay, the following Delay and Corresponding Gain Vectors are formulated.

Table:1 Different variation of delays and gain of LOS with Multipath are shown below with 4 sequential Taps.

\begin{tabular}{|l|c|c|c|}
\hline \multicolumn{1}{|c|}{ Path \# } & Cases & Delay & Power \\
\hline & & (ns) & (Db) \\
\hline 1. (LOS) & & 0 & 0 \\
\hline 2. (Multipath) & Case-1 & 6 & -0.5 \\
\hline 3. (Multipath) & Case-2 & 5 & -0.5 \\
\hline 4. (Multipath) & Case-3 & 4 & -0.5 \\
\hline 5. (Multipath) & Case-4 & 3 & -0.5 \\
\hline
\end{tabular}


The LOS and Multipath are up-converted through Vector Signal Generator (VSG) where we have set the RF Carrier as $1.45 \mathrm{GHz}$ and a Transmit Power of 5dBm. The Vector Signal Analyzer (VSA) is accordingly tuned to $1.45 \mathrm{GHz}$ with a span of $410 \mathrm{MHz}$. The Cross-Correlated data between Reference signal and Received LOS with Multipath is obtained through VSA. Then the VSA Cross-Correlation trace data is captured into MATLAB Simulink and further processing is done to resolute the overlapped LOS and Multipath.

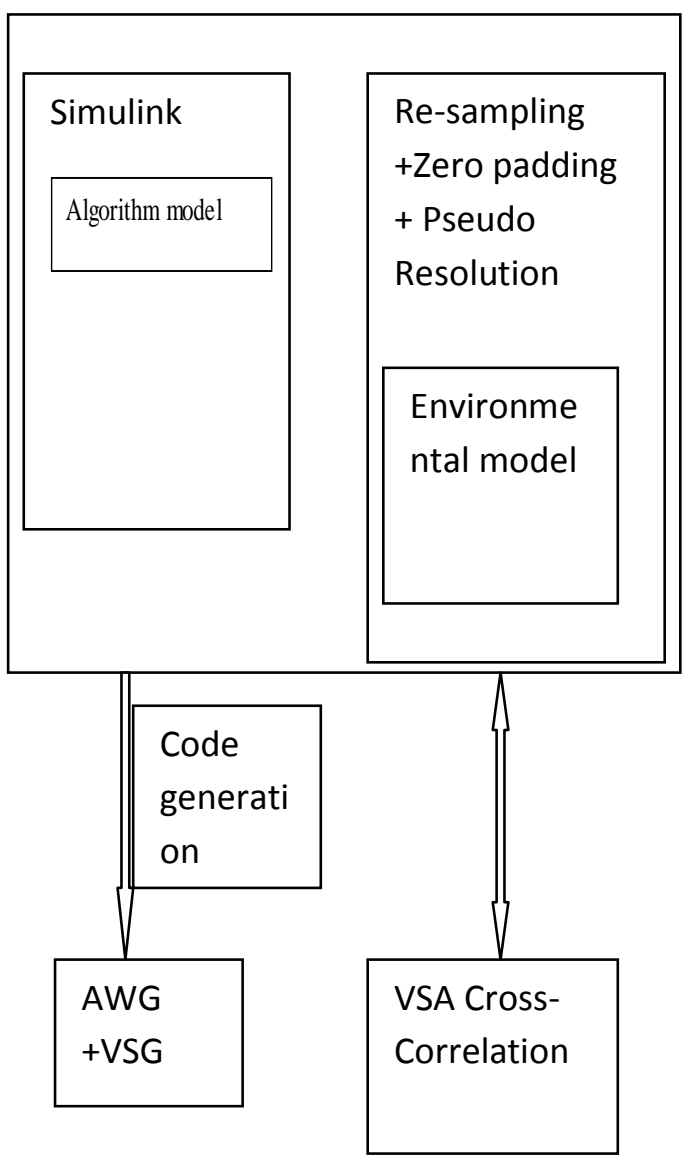

Fig 1. experimental Setup

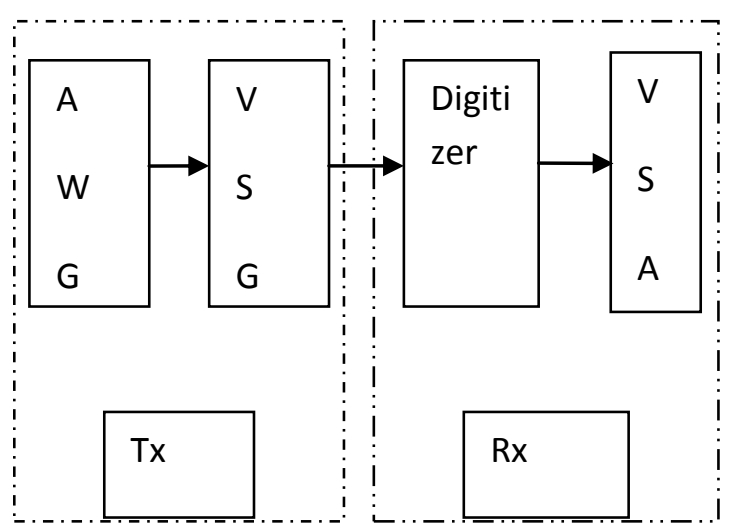

Fig 2 Block diagram of experimental setup

\section{MATHEMATICAL EXPRESSION FOR THE DESIGN OF THE SYSTEM}

\subsection{Radar Range \& Cross Range}

The range of the target can be computed by measuring the time $\Delta \mathrm{t}$ traveled by pulse to cover the two-way path between the radar and the target.[9]. As we know that electromagnetic waves travel at the speed of light, then

$\mathrm{R}=\mathrm{c} \Delta \mathrm{t} / 2$

Where $\mathrm{R}$ is in meters and $\Delta \mathrm{t}$ is in seconds. For the two-way time delay the factor of $1 / 2$ is needed.

Range resolution, denoted as $\Delta \mathrm{R}$, which gives a close proximity of distinct targets as distinct objects. [10] There are mainly two range at which radar operate i.e. between a minimum range Rmin, and maximum range Rmax. [11] The distance between $R \max$ and $R \min$ is divided into $M$ range bins, each of width $\Delta R$,

$\mathrm{M}=\left(\mathrm{R}_{\mathrm{MAX}}-\mathrm{R}_{\mathrm{MIN}}\right) / \Delta \mathrm{R}$

Targets which are separated by at least can be resolved in range and when the targets which are within the same range cell can be resolved in cross range (i.e. azimuth). Let us consider that two targets that are localized at ranges R1 and $\mathrm{R} 2$ with time delays $\mathrm{t} 1$ and $\mathrm{t} 2$, respectively. [12] The difference between those two ranges is given by:

$\Delta \mathrm{R}=\mathrm{R}_{2}-\mathrm{R}_{1}=\mathrm{c}\left(\mathrm{t}_{2}-\mathrm{t}_{1}\right) / 2=\mathrm{c} \partial \mathrm{t} / 2$

To find the minimum $\Delta \mathrm{R}$, let us consider that two targets are separated by distance ct/4, where $t$ is the pulse width. In this case, when the pulse trailing edge strikes target 2 the leading edge would have traveled backwards a distance ct, and the returned pulse would be composed of returns from both targets (i.e., unresolved return). However, if the two targets are at least ct/2 apart, then as the pulse trailing edge strikes the first target the leading edge will start to return from target 2 , and two distinct returned pulses will be produced. [13]Thus, $\mathrm{dR}$ should be greater or equal to $\mathrm{ct} / 2$. And since the radar bandwidth $B$ is equal to $1 / t$, then

$\Delta \mathrm{R}=\mathrm{c} \tau / 2=\mathrm{c} / 2 \mathrm{~B}$

Maximum radar range $\mathrm{R}_{\text {max }}$ is given by

$\mathrm{R}_{\max }=\left(\mathrm{P}_{\mathrm{t}} \mathrm{G}^{2} \lambda^{2} \sigma /(4 \Pi)^{3} \mathrm{~S}_{\mathrm{MIN}}\right)^{1 / 4}$

Where Smin denotes the minimum detectable signal power. The target RCS can be defined by received intensity of the backscattered energy that has the same polarization as the radars receiving antenna.[14]Power density of a wave incident on a target located at range away from the radar is $\mathrm{P}_{\mathrm{Di}}$. The amount of reflected power from the target is

$\operatorname{Pr}=\sigma \mathrm{P}_{\mathrm{Di}}$

$\sigma$ denotes the target cross section.

The total target scattered RCS [15] is given by

$\sigma_{\mathrm{i}}=(1 / 4 \Pi) \int_{\varphi \mathrm{s}=0} \int_{\Theta \mathrm{s}=0}=\sigma\left(\Theta_{\mathrm{S}}, \varphi_{\mathrm{s}}\right) \sin \left(\Theta_{\mathrm{s}}\right) \mathrm{d} \Theta \mathrm{d} \varphi_{\mathrm{s}}$

Radar Cross Section which depends on aspect angle is given by

$\operatorname{RCS}=\lambda / 2 * \sin (\theta)$ 


\subsection{Sampling}

A discrete time sequence $x[n]$ is generated by sampling [16] a continuous time signal $\mathrm{x}(\mathrm{t})$ at uniform intervals

$\mathrm{x}[\mathrm{n}]=\mathrm{x}(\mathrm{t}) \mid \mathrm{t}=\mathrm{nT}=\mathrm{x}(\mathrm{nT}) \quad \mathrm{n}=-2,-1,0,1,2 \ldots$.

Here time variable $t$ of the continuous-time signal is related to the time variable $\mathrm{n}$ of the discrete-time signal at discrete-time instant $t$ is given by

$\mathrm{t}=\mathrm{nT}=\mathrm{n} / \mathrm{F}=2 * \Pi * \mathrm{n} / \mathrm{w}$

Where $\mathrm{F}=1 / \mathrm{T}$ denoting sampling frequency and $\mathrm{w}=2 * \Pi * \mathrm{~F}$ denoting the sampling angular frequency

\subsection{Cross-Correlation}

Correlation relates to the degree to which the signal at the present time reflects its values in the past. Correlation [17] of a signal with another signal is known as Cross correlation. Used to detect and identify similarities in signals and delays in systems

$\mathrm{R}_{\mathrm{xy}}(\tau)=\mathrm{E}[\mathrm{x}(\mathrm{t}) \mathrm{y}(\mathrm{t}+\tau)]$

$R_{x y}$ denotes the cross-correlated signal.

Where $x(t)$ and $y(t)$ are the two signals (t) is the reference signal and $y(t)$ is the received signal.

\subsection{IFFT}

The IFFT [18][19] of the signal can be expressed as

$$
\begin{array}{ll}
\mathrm{X}[\mathrm{k}]=\sum_{\mathrm{n}=0}^{\mathrm{N}-1} \mathrm{X}[\mathrm{n}] \mathrm{W}^{\mathrm{kn}}{ }_{\mathrm{N}} & 0 \leq \mathrm{k} \leq \mathrm{N}-1 \\
\mathrm{x}[\mathrm{n}]=1 / \mathrm{N}\left(\Sigma_{\mathrm{k}=0}^{\mathrm{N}-1} \mathrm{X}[\mathrm{n}] \mathrm{W}^{-\mathrm{kn}}{ }_{\mathrm{N}}\right) & 0 \leq \mathrm{n} \leq \mathrm{N}-1
\end{array}
$$

\subsection{Justification of choosing multipath delays $6 \mathrm{~ns}$, 5ns, 4ns, 3ns}

The following Fig.-1 shows the Multipath phenomenon. So, to mitigate such multipath effects a prior calculation is done regarding the probability of multipath occurrence in terms of delay. Depending upon the antenna height, target height, target range, the excess path delay has been evaluated.
Calculations of Multipath:

Let us consider some of parameters for the calculation of Multipath

$\mathrm{ht}=$ antenna height $=10$ meter;

Target height $=10$ meter;

Target range $=\mathrm{AF}=\mathrm{EB}=2 \mathrm{~km}$;

Angle $\mathrm{CAF}=7.5$ degree;

Angle $\mathrm{CAE}=82.5$ degree;

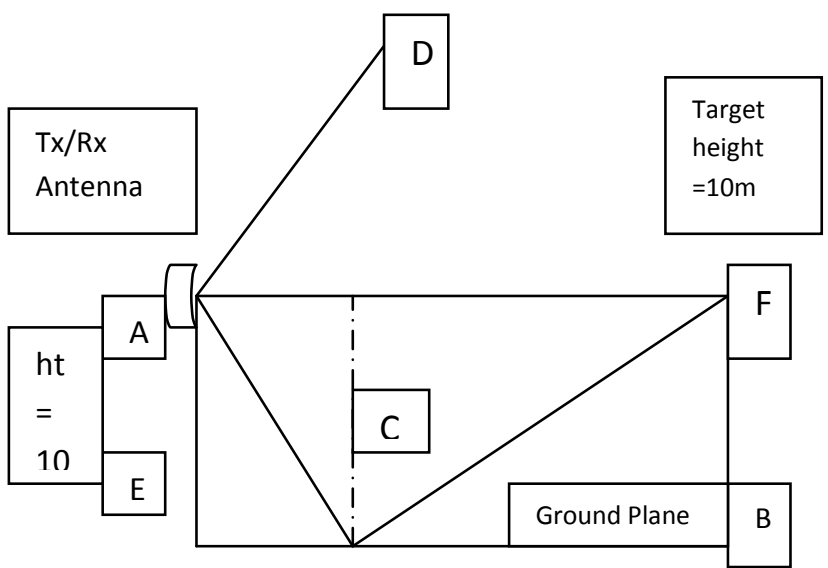

Fig.-3 Multipath model

Hence,

$\tan (\mathrm{CAE})=\mathrm{EC} / \mathrm{AE}=7.595$

Therefore

$\mathrm{BC}=10 * 7.595$ meter $=75.95$ meter.

According to Fig - :

$\mathrm{CB}=\mathrm{EB}-\mathrm{EC}=2000-75.95=1924.05$ meter.

$\sin (\mathrm{CAE})=\mathrm{EC} / \mathrm{AC}$

Therefore

$\mathrm{AC}=\mathrm{EC} / 0.991=76.63$ meter

Now, $\mathrm{CF} 2=\mathrm{FB} 2+\mathrm{CB} 2$;

Then $\mathrm{CD}=1924.30$

Hence Excess path $=(\mathrm{AC}+\mathrm{CF})-\mathrm{AF}=0.93$ meter.

$\&$ Excess delay $=3.1 \mathrm{~ns}$

As per the above calculation, we can find the reason behind choosing the Multipath of $6 \mathrm{~ns}, 5 \mathrm{~ns}, 4 \mathrm{~ns}$ and in worst case as 3ns sequentially.

\section{RESULTS}

\subsection{Case-1: For 6ns delay between LOS and Multipath: RF Carrier $=1.45$ GHz; System}

\section{Bandwidth = $400 \mathrm{MHz}$}

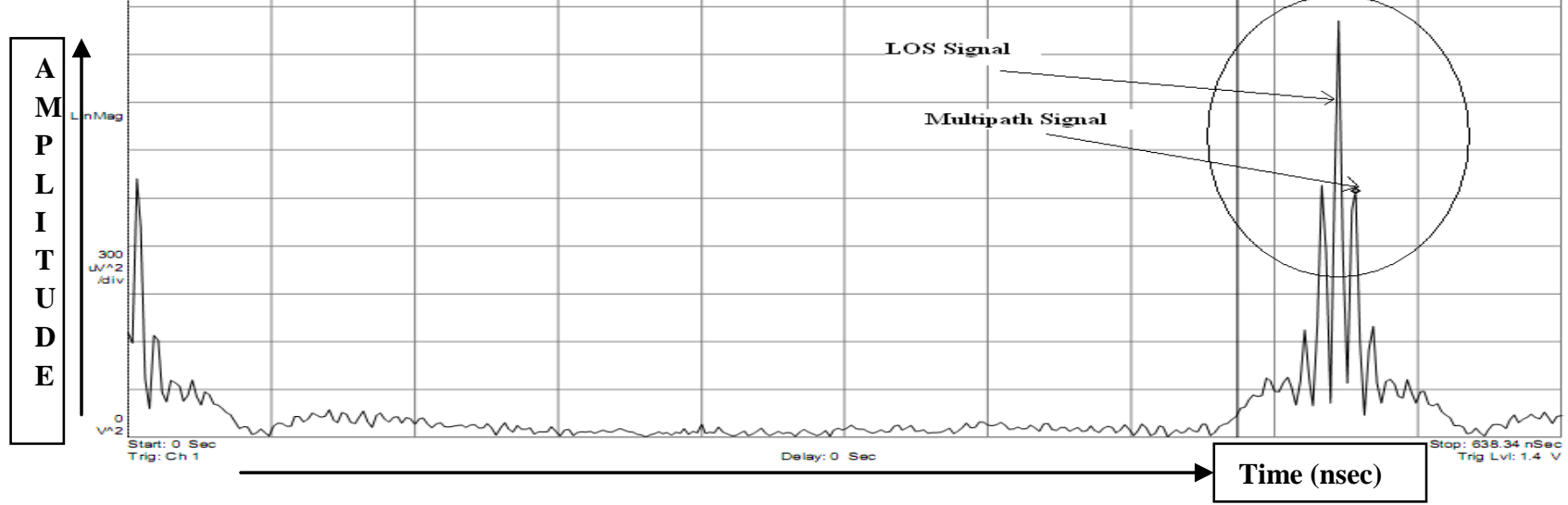

Fig .4a.Received signal observed in VSA (after Cross Correlation) 


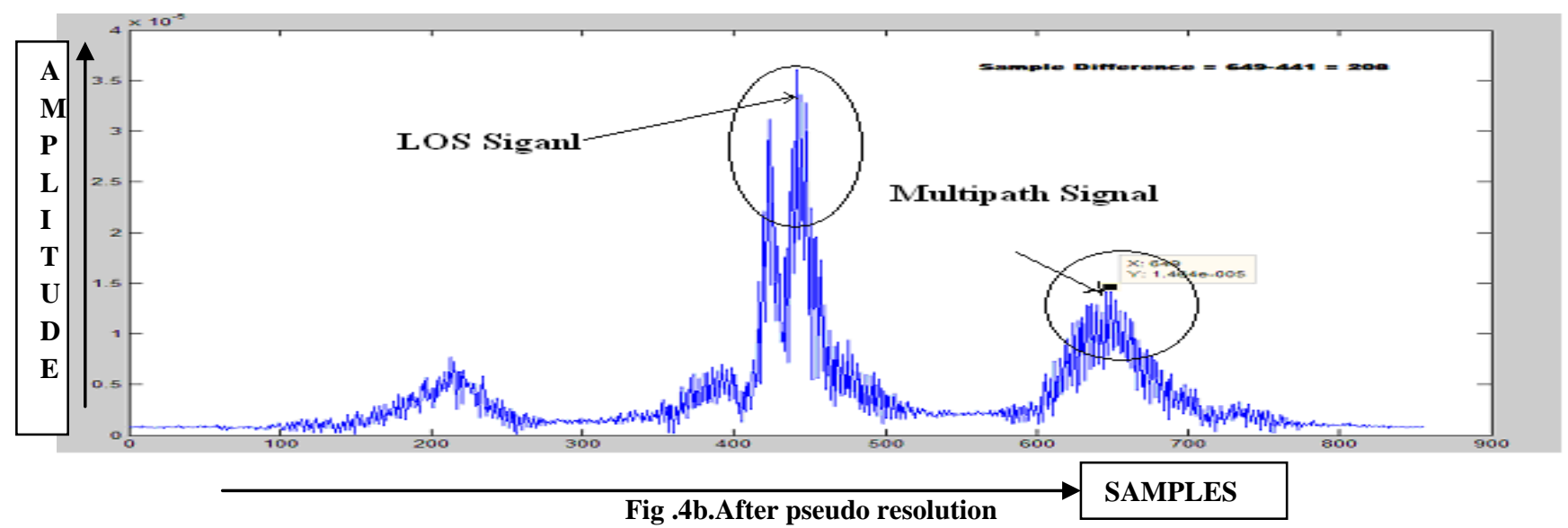

\subsection{Case-1: For 5ns delay between LOS and Multipath: RF Carrier = 1.45 GHz; System Bandwidth = $400 \mathrm{MHz}$}
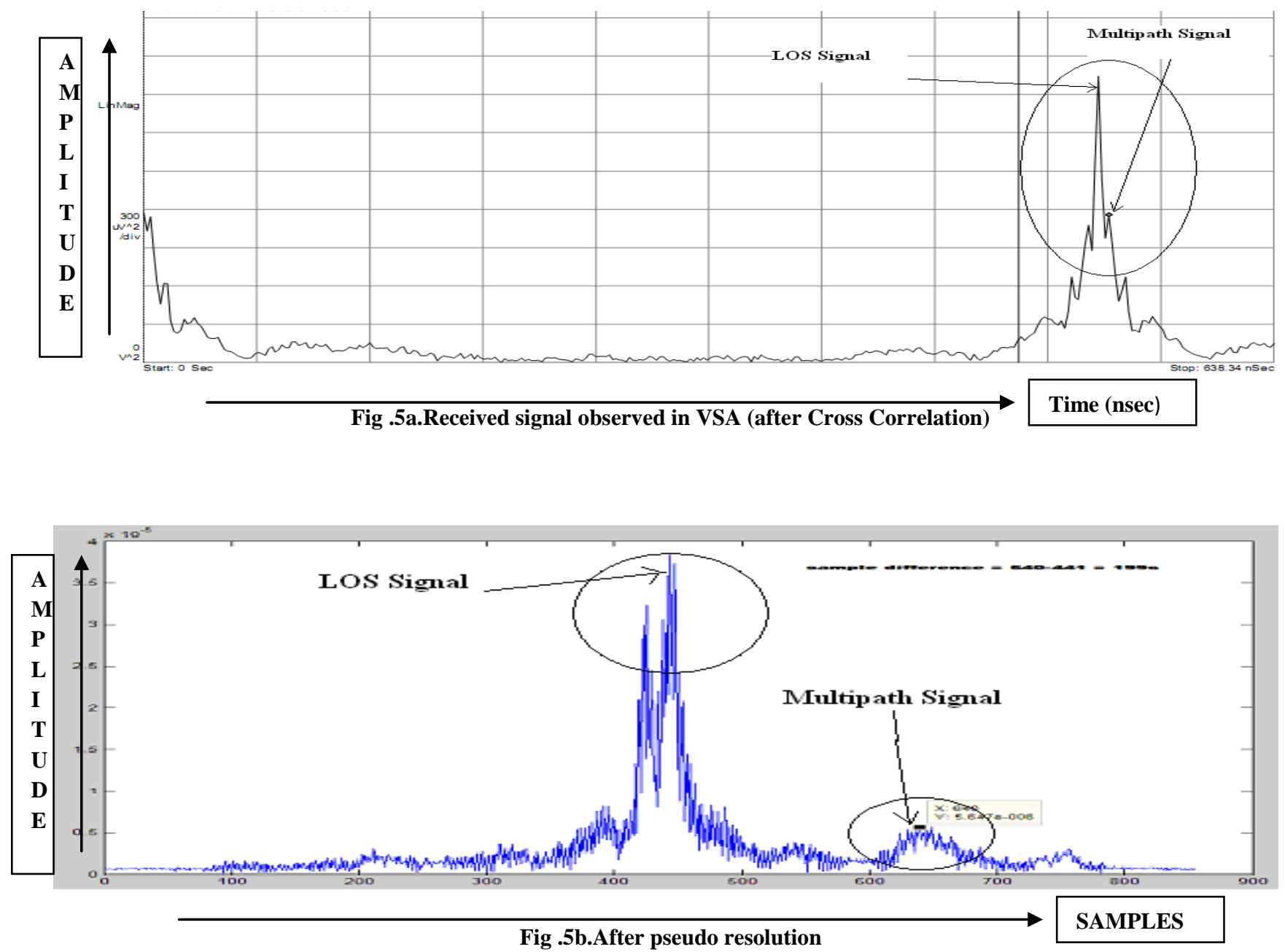
4.3 Case-1: For 4ns delay between LOS and Multipath: RF Carrier = 1.45 GHz; System Bandwidth $=400 \mathrm{MHz}$
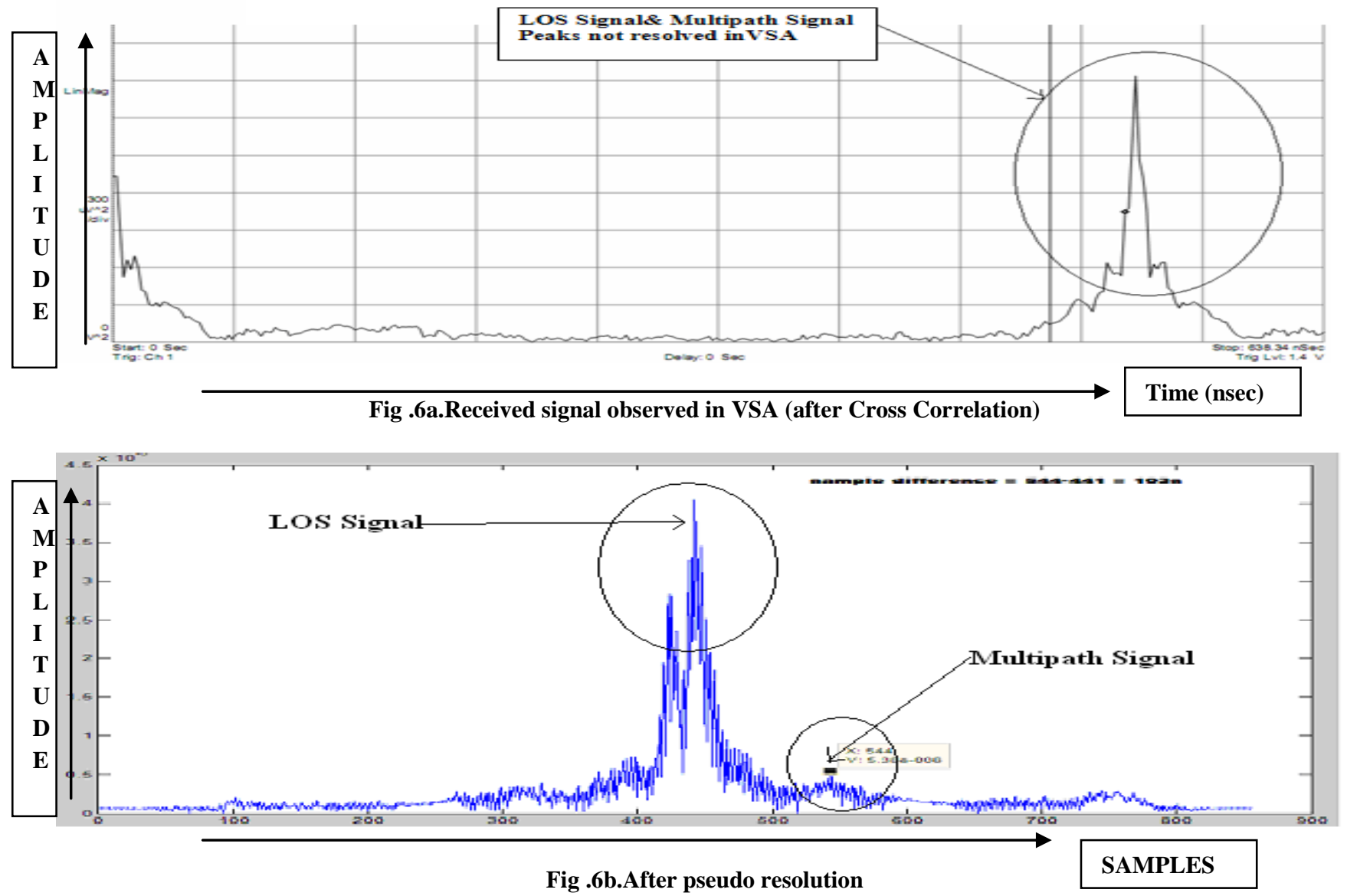

4.4 Case-1: For 3ns delay between LOS and Multipath: RF Carrier = 1.45 GHz; System Bandwidth $=400 \mathrm{MHz}$

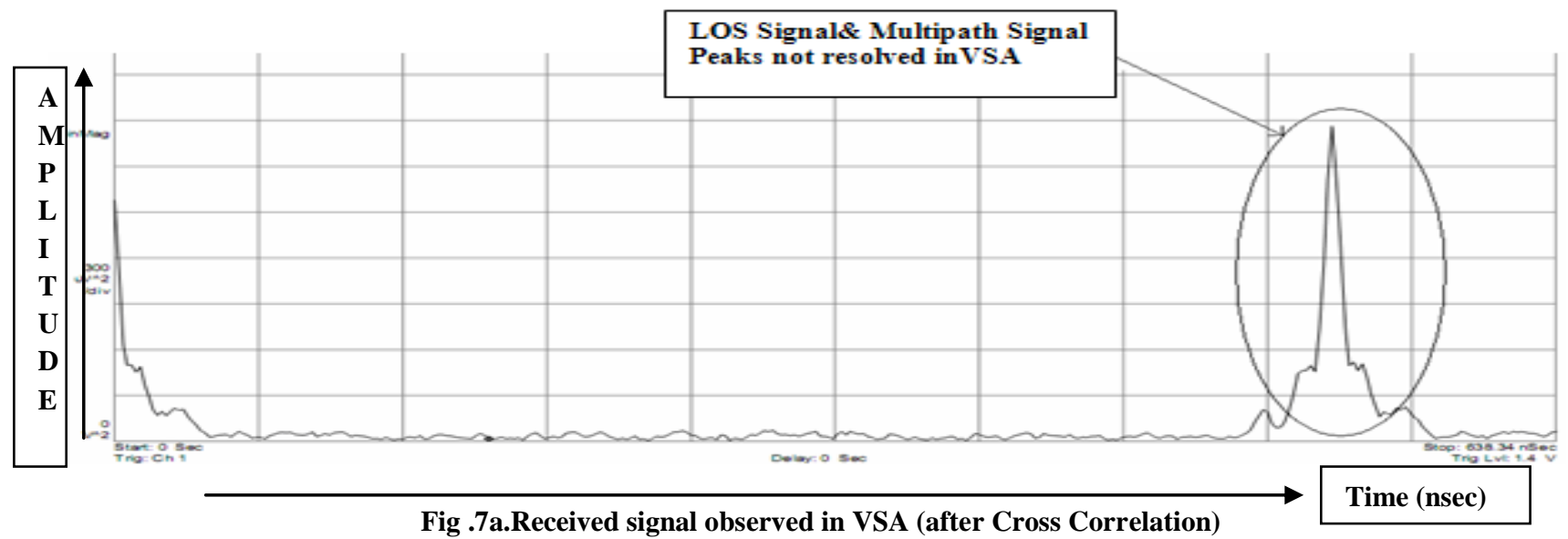




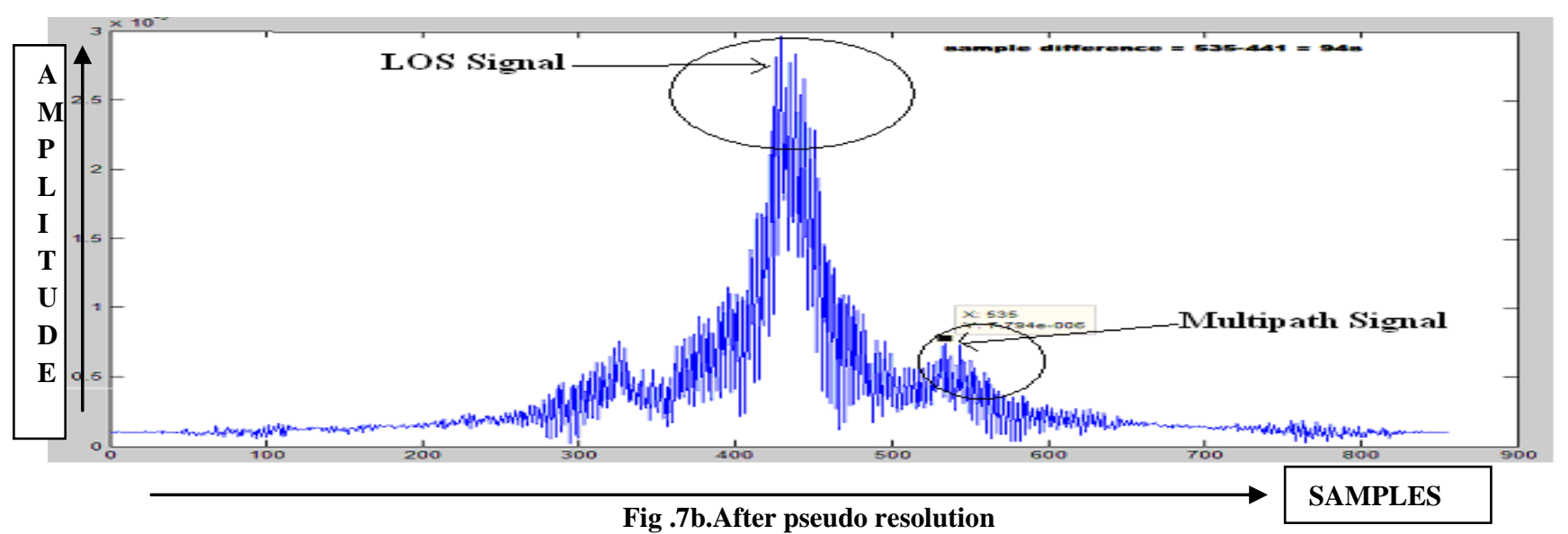

In cases of $6 \mathrm{~ns}, 5 \mathrm{~ns}, 4 \mathrm{~ns}$, 3ns delay between LOS signal and Multipath signal peaks are very difficultly resolved in VSA as referred in Fig 4a, 5a, 6a \& 7a So to Discriminate LOS and multipath signal the method of pseudo-resolution is used. In this method, the received signal at the receiver is re-sampled and then its virtual bandwidth is accordingly increased by stuffing a set of zeros (i.e., 688 zeros) at the end of the received cross-correlated frame coming from VSA. Thus a frame of [1024 x 1] is generated and being again resampled with a higher rate as the pseudo bandwidth is more after appending zeros. Then this zero padded frame is processed through IFFT routine. Using this technique both LOS and Multipath signal is discriminated as shown in Fig $\mathbf{4 b}, \mathbf{5 b}, \mathbf{6 b}$ \& $7 \mathbf{b}$.

\section{CONCLUSION}

From this experiment we see that where the VSA fails to resolute the LOS \& multipath, the method of pseudo resolution becomes necessarily to be adopted. The experimental result proves this fact. The LOS \& multipath are precisely distinguished by the use of pseudo-resolution method. We also show that by increasing bandwidth it becomes easier for the signal to be resolute at the receiver end.

\section{REFERENCES}

[1] M. I. Skolnik, Introduction to Radar Systems, second edition, McGraw Hill publications, 1980..

[2] Radar basic description is online available at: http://en.wikipedia.org/wiki/radar

[3] Barrientos et al.," CAWAS: Collision Avoidance and Warning system for Automotives based on Satellite ",Proceedings of the $8^{\text {th }}$ International IEEE Conference on Intelligent Transportation Systems Vienna, Austria, September 13-16, 2005

[4] Xin Huang "Smart Antennas for Intelligent Transportation Systems", 6th International Conference on ITS Telecommunications Proceedings, 2006.

[5] P.Soma, L.C.Ong, and Y.W.M.Chia, "Effects of down tilt antenna on the Multipath propagation characteristics in the presence of an imperfect obstacle,"in Proc. IEEE Asia Pacific Microwave Conference, vol.3, Dec. 1999,pp. 610-613.
[6] Multipath as a target representation is online available at: http://en.wikipedia.org/wiki/multipath

[7] Ashcraft, I.S. and Long, D.G.(2005). Observation and Characterization of Radar Backscatter Over Greenland. IEEE Transactionson Geoscience and Remote Sensing, 43(2):225-237.

[8] A.R.Brenner, "Polyphase Barker sequences up to length 45 with small alphabets" IEE electronic letter, June 1998 pp. $1576 \mathrm{C} 1577$

[9] 'Introduction to radar system' by Merrill I skolnik $2^{\text {nd }}$ edition, the McGraw Hill company

[10] W.C.Carrar, R.G.Goodman, R.M.Majewski, Spotlight Synthetic Aperture Radar: Signal Processing Algorithms, Artech House, Boston, 1995.

[11] 'synthetic aperture radar, system and signal processing' by john c. curlander Robert n.mc donough, wiley series in remote sensing, a wiley -Inter science publication

[12] D.R.Wehner, High-Resolution Radar, second edition, Artech House ,Norwood, MA,1995.

[13] P.Bidigare, T.Stevens, B.Correll and M.Beauvais, "Minimum Radar Cross Section Bounds for Passive Radar Responsive Tags", Signals, Systems and Computers, Nov 2004, Vol. 2, pp.1441C1445.

[14] Barton, D.k.: "Radar system analysis" originally published by prentice-hall in 1964 and republished by artech house, norwood, mass., in 1977

[15] Radar range and cross range in 'Radar system analysis and design using matlab" by bassem $r$ mahafza,colsa corporation, Huntsville, Alabama ,chapman \& hall/crc

[16] Crochiere, R.and Rabiner, L., "Interpolation \& decimation of digital signals-a tutorial review," Proceedings of the IEEE 69(3), 300-331(1981).

[17] Turin, Geroge L "An introduction to matched filers" IRE transactions on Information theory June 1960, pp. $311 \mathrm{C} 329$.

[18] The Mathworks, Inc, MATLAB Documentation: Discrete Fourier Transform. Version7.9.0.529 (R2009b).

[19] J.J.Duistermaat, Fourier Integral Operators, Birkhauser, Boston, 1 